\title{
Selection of surgical procedures and analysis of prognostic factors in patients with primary gastric tumour based on Cox regression: a SEER database analysis based on data mining
}

\author{
Cheng X $u^{1}$, Qingling Chen ${ }^{1}$, Fan Ye' ${ }^{2}$, Qi Fan ${ }^{1}$, Qing Wang ${ }^{1}$ \\ ${ }^{1}$ College of Computer Science and Technology, Huaibei Normal University, Huaibei, China \\ ${ }^{2}$ School of Business, Macau University of Science and Technology, Macau, China
}

Gastroenterology Rev 2021; 16 (2): 144-154

DOI: https://doi.org/10.5114/pg.2021.106666

Key words: gastric cancer, SEER, surgical method, prognosis.

Address for correspondence: Prof. Qi Fan, College of Computer Science and Technology, Huaibei Normal University, Huaibei 235000, China, e-mail: fanqimai@hotmail.com

\begin{abstract}
Introduction: There are numerous types of surgery for patients with primary gastric tumour, which can be summarized as radical surgery or palliative surgery. Different surgical procedures will have further effects for different stage of patients.

Aim: We will use the resources of the SEER database (2010-2015) to explore the therapeutic value of surgery and prognostic factors.

Material and methods: Kaplan-Meier analysis/log-rank testing for data analysis and multivariate analysis was conducted through a Cox proportional model.

Results: Fourteen thousand five hundred and seven cases of primary gastric tumours identified in the period from 2010 to 2015. In a multivariate cox regression analysis, the following factors were associated with better primary gastric patients survival (Surgical method, Age at diagnosis, histological grade). Through Kaplan-Meier analysis $(p<0.005)$ we also found that for the patient group the survival rate of using gastrectomy (partial, subtotal, hemi-) surgery is the lowest.

Conclusions: Among patients with multivariate Cox regression model, type of surgery, age at diagnosis, and histological grade were the top 3 factors affecting patient survival. In palliative surgery, laser excision is the best surgical method of local tumour excision, and the survival of patients of this group is obviously better than in other groups. In radical surgery, near-total gastrectomy and radical gastrectomy, in continuity with the resection of other organs, are better surgical methods, while gastrectomy (partial, subtotal, hemi-) is the worst type of surgery in terms of prognosis, and even the survival rate in the later stage (after 3 years) is worse than in the group without surgery.
\end{abstract}

\section{Introduction}

Gastric cancer is the fifth most common cancer worldwide. It is the fourth most commonly occurring cancer in men and the seventh most commonly occurring cancer in women. There were over 1 million new cases in 2018 [1]. In the past 60 years, the incidence of gastric cancer has decreased significantly compared with previous data. But it is undeniable that gastric cancer is still a very serious disease and that it is a very serious problem; the cause of gastric cancer is not very clear, but there is no doubt that some people are more likely to suffer from this disease than others [2].
The most commonly used staging system for gastric cancer is the Tumor/Node/Metastasis (TNM)/American Joint Committee on Cancer (AJCC) system. Successive editions of this staging system have been released reflecting the advances in our knowledge of gastric cancer prognosis and treatment approaches [3]. The staging systems we used in this study are AJCC TNM, $7^{\text {th }}$ ed. (2010-2015) and AJCC Stage Group, $7^{\text {th }}$ ed. (2010-2015).

\section{Aim}

In order to explore the cause of gastric cancer, we analysed the data from the SEER database and obtained some valuable information. The contents of our study 
are as follows: the effects of various physical indicators on the incidence of gastric cancer, the differences of different type of surgery for patients with different stages, and the differences of the different type of surgery for the same operation.

\section{Material and methods}

The cases evaluated in this analysis were extracted from the SEER-18 registry [4]; in order to achieve this, SEER ${ }^{\star}$ Stat software (Version 8.3.5) was used. The date of the SEER data submission was November 2018.

\section{Selection of the study cohort}

Patients were screened from the SEER database between 2010 and 2015. In order to identify patients with primary gastric tumours, "Stomach" in the CS Schema-AJCC 6th Edition category was selected. Patients with primary tumours are identified by screening for "Yes" in the attribute first malignant primary indicator. Cases with deficient information about their marital status at diagnosis, insurance recode (2007+), grade, RX Summ-SurgPrimSite (1998+), CS tumour size (20042015), AJCC $7^{\text {th }}$ stage, and survival were excluded.

\section{Data collection}

Information extracted for each patient included sex, race recode $(\mathrm{W}, \mathrm{B}, \mathrm{Al}, \mathrm{API})$, age at diagnosis, marital status at diagnosis, insurance recode $(2007+)$, grade, RX Summ--Surg Prim Site (1998+), CS tumour size (2004-2015), first malignant primary indicator, survival (months), vital status recode (study cut-off used), $7^{\text {th }}$ edition $T, N$, and $M$ stages, and AJCC $7^{\text {th }}$ edition stage group.

\section{Statistical considerations}

In this study, Kaplan-Meier analysis and log-rank testing were used for survival comparisons (including both overall survival and staging survival) according to the AJCC pathological stage. The cox proportional hazard model was conducted to produce multivariate analyses. A score of $77.6 \%$ is obtained by C-index calculation of the Cox model, and the score of the AUC is $77.0 \%$, which shows that the model is statistically significant. All statistical analyses were performed in Python (Python3.7) and $\mathrm{R}$ language (R version 3.6.3).

\section{Results}

\section{Patients' characteristics}

A total of 10,050 patients with surgically treated primary gastric tumour were identified in the period from 2010 to 2015 and were included in the analysis (Figure 1). In the dataset, gastrectomy (partial, subtotal, hemi-) rep- resented half of the cases (50.5\%), local tumour destruction $(0.1 \%)$, local tumour excision (7.3\%), near-total or total gastrectomy (11.6\%), gastrectomy with removal of a portion of the oesophagus ( $N=18.9 \%)$, gastrectomy with resection in continuity with the resection of other organs (10.9\%), surgery NOS (0.4\%), and gastrectomy NOS (0.3\%). The most frequent age group was 40-69 years $(56.3 \%)$, followed by the group of $>69$ years (39.9\%), and the age group < 40 years (3.8\%). White race comprised $66.1 \%$, black race represented $13.7 \%$, Asian or Pacific Islander racial groups represented $19.5 \%$, and the American Indian/Alaska Native racial groups represented $0.7 \%$. The distribution of patients according to AJCC stages and type of surgery is shown in Table I.

\section{Multivariable prediction model based on cox regression model}

The data were analysed by the cox regression mod$\mathrm{el}$, and the prediction model with the reference value was obtained (C-index score 77.6\%, AUC score 77.0\%). Some conclusions can be drawn from the nomogram of the cox regression model (Figure 2). It can be concluded from the calibration curve (Figure 3 ) that the age of diagnosis, histological grade, and the type of surgery have a great impact on the survival rate of patients. In the following, we will study the choice of surgical type.

Multivariate analysis for factors affecting primary gastric cancer survival was conducted (evaluating the following factors: sex, race, age at diagnosis, marital

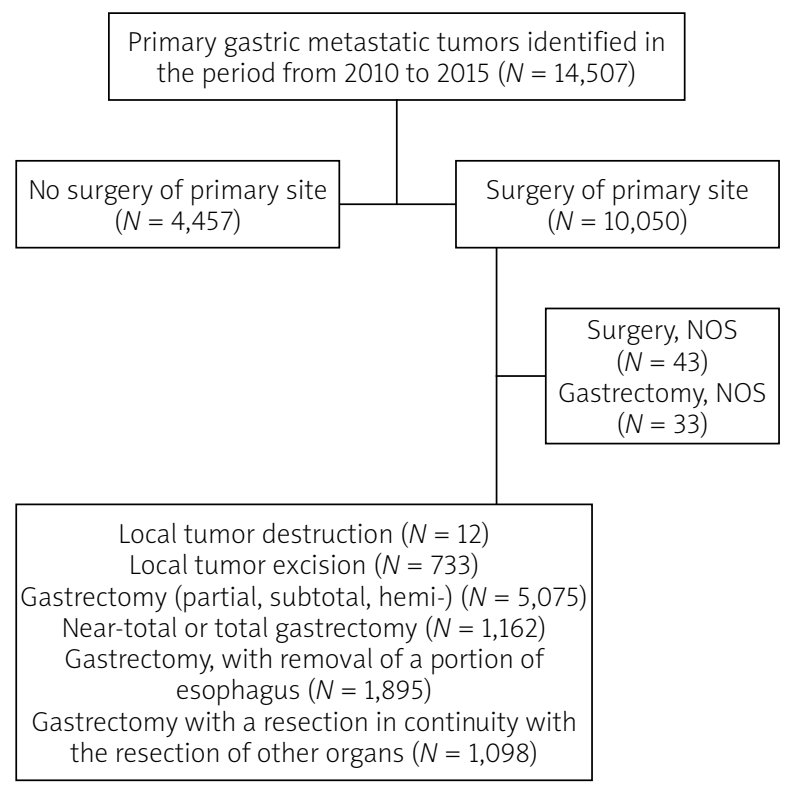

Figure 1. Study enrolment of 14,507 primary gastric cancer records present 10,050 patients who had had surgery were included in the analysis 
Table I. Baseline characteristics of included patients in the cohort $(N=10,050)$

\begin{tabular}{|c|c|}
\hline Parameter & $N(\%)$ \\
\hline \multicolumn{2}{|l|}{ Age [years]: } \\
\hline$<40$ & $379(3.8)$ \\
\hline $40-69$ & $5657(56.3)$ \\
\hline$>69$ & $4014(39.9)$ \\
\hline \multicolumn{2}{|l|}{ Race: } \\
\hline White & $6641(66.1)$ \\
\hline Black & $1381(13.7)$ \\
\hline Asian or Pacific Islander & $1959(19.5)$ \\
\hline American Indian/Alaska Native & $69(0.7)$ \\
\hline \multicolumn{2}{|l|}{ Gender: } \\
\hline Female & $3980(39.6)$ \\
\hline Male & $6070(60.4)$ \\
\hline \multicolumn{2}{|l|}{ Insurance recode: } \\
\hline Yes & $3593(35.8)$ \\
\hline No & $6457(64.2)$ \\
\hline \multicolumn{2}{|l|}{ Histological grade: } \\
\hline Well differentiated; Grade I & $1427(14.2)$ \\
\hline Moderately differentiated; Grade II & $2814(28.0)$ \\
\hline Poorly differentiated; Grade III & $5474(54.5)$ \\
\hline Undifferentiated; anaplastic; Grade IV & $335(3.3)$ \\
\hline \multicolumn{2}{|l|}{ Type of surgery: } \\
\hline Local tumour destruction & $12(0.1)$ \\
\hline Local tumour excision & $733(7.3)$ \\
\hline Gastrectomy (partial, subtotal, hemi-) & $5075(50.5)$ \\
\hline Near-total or total gastrectomy & $1162(11.6)$ \\
\hline $\begin{array}{l}\text { Gastrectomy, with removal of a portion of } \\
\text { oesophagus }\end{array}$ & $1895(18.9)$ \\
\hline $\begin{array}{l}\text { Gastrectomy with a resection in continuity } \\
\text { with the resection of other organs }\end{array}$ & $1098(10.9)$ \\
\hline Surgery NOS & $43(0.4)$ \\
\hline Gastrectomy NOS & $33(0.3)$ \\
\hline \multicolumn{2}{|l|}{ Pathological AJCC stage: } \\
\hline 0 & $34(0.3)$ \\
\hline 1 & $305(3.0)$ \\
\hline $\mathrm{IA}$ & $1873(18.6)$ \\
\hline IB & $929(9.2)$ \\
\hline II & $131(1.3)$ \\
\hline$\| \mathrm{A}$ & $1038(10.3)$ \\
\hline$\| \mathrm{B}$ & $1226(12.2)$ \\
\hline IIIA & $1231(12.2)$ \\
\hline IIIB & $1228(12.2)$ \\
\hline IIIC & 1049 (10.4) \\
\hline IIINOS & $2(0.02)$ \\
\hline IV & $868(8.6)$ \\
\hline UNK Stage & $136(1.4)$ \\
\hline
\end{tabular}

status at diagnosis, insurance recode, grade, surgery, tumour size, survival months, T stage, $N$ stage, $M$ stage, and type of surgery). The following factors were associated with better primary gastric cancer survival (sex, race, age at diagnosis, marital status at diagnosis, insurance recode, grade, surgery, survival months, T stage, $\mathrm{N}$ stage, $M$ stage, and type of surgery) $(p<0.005)$ (Figure 4).

\section{Survival outcomes according to AJCC $7^{\text {th }}$ ed. and type of surgery}

Overall, primary gastric cancer survival was compared according to both AJCC $7^{\text {th }}$ ed. and type of surgery. Log-rank testing with pairwise comparisons between all different stages was conducted. In all the stage groups, except for the comparison between stages $0, \mathrm{I}, \mathrm{II}$, and III NOS (Figures $5 \mathrm{~A}, \mathrm{~B}, \mathrm{E}, \mathrm{K}$ ) there are significant $(p<0.005)$ differences among different stages. Part of the legend is shown in Figure 5. From the results of the Kaplan-Meier analysis, it can be seen that in the 0 stage group, the survival of patients who received gastrectomy was better, and in patients who received local tumour excision it was the worst (Figure $5 \mathrm{~A}$ ). The same thing happened in the I stage group (Figure $5 \mathrm{~B}$ ). In the staging group except 0 , I, IIINOS and IV stage group (Figures $5 \mathrm{C}-\mathrm{J}$ ), the survival of patients receiving gastronomy (partial, subtotal, hemi-) was the worst, while the survival of patients who received local tumour destruction and local tumour excision was the best. In the NOS III and IV stage groups (Figures $5 \mathrm{~K}, \mathrm{~L}$ ), the survival was the worst among patients who did not undergo surgery.

It is noteworthy that due to the small amount of data in the 0 group $(0.3 \%$, Figure $5 \mathrm{~A})$ and the III NOS group $(0.02 \%$, Figure $5 \mathrm{~K})$ their importance is not high, thus affecting the overall value. More detailed studies on how surgical methods affect survival benefits will be described in later sections.

\section{Survival outcomes according to the type of surgery and surgical methods}

There was no significant difference among different groups except the local tumour destruction group $(p=0.77$, Figure $6 \mathrm{~B})$ and local tumour excision group ( $p=0.29$, Figure $6 \mathrm{C})$, but there was a significant difference in other groups $(p<0.005)$. In the 3 -year survival, the survival among the "no surgery of primary site" group was the worst, but that of the gastrectomy (partial, subtotal, hemi-) group was the worst when it was greater than the 3-year survival (Figure $6 \mathrm{~A}$ ). For the patients who had local tumour destruction surgery, the survival of each surgical method was roughly the same (Figure $6 \mathrm{~B}$ ). In the local tumour excision surgery, the survival of the laser excision group was 


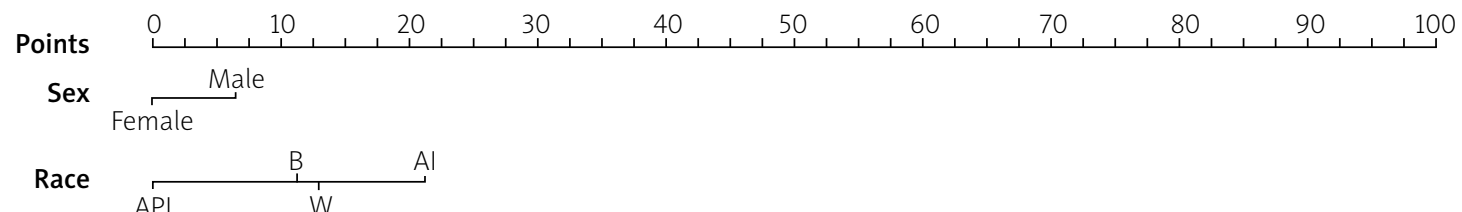

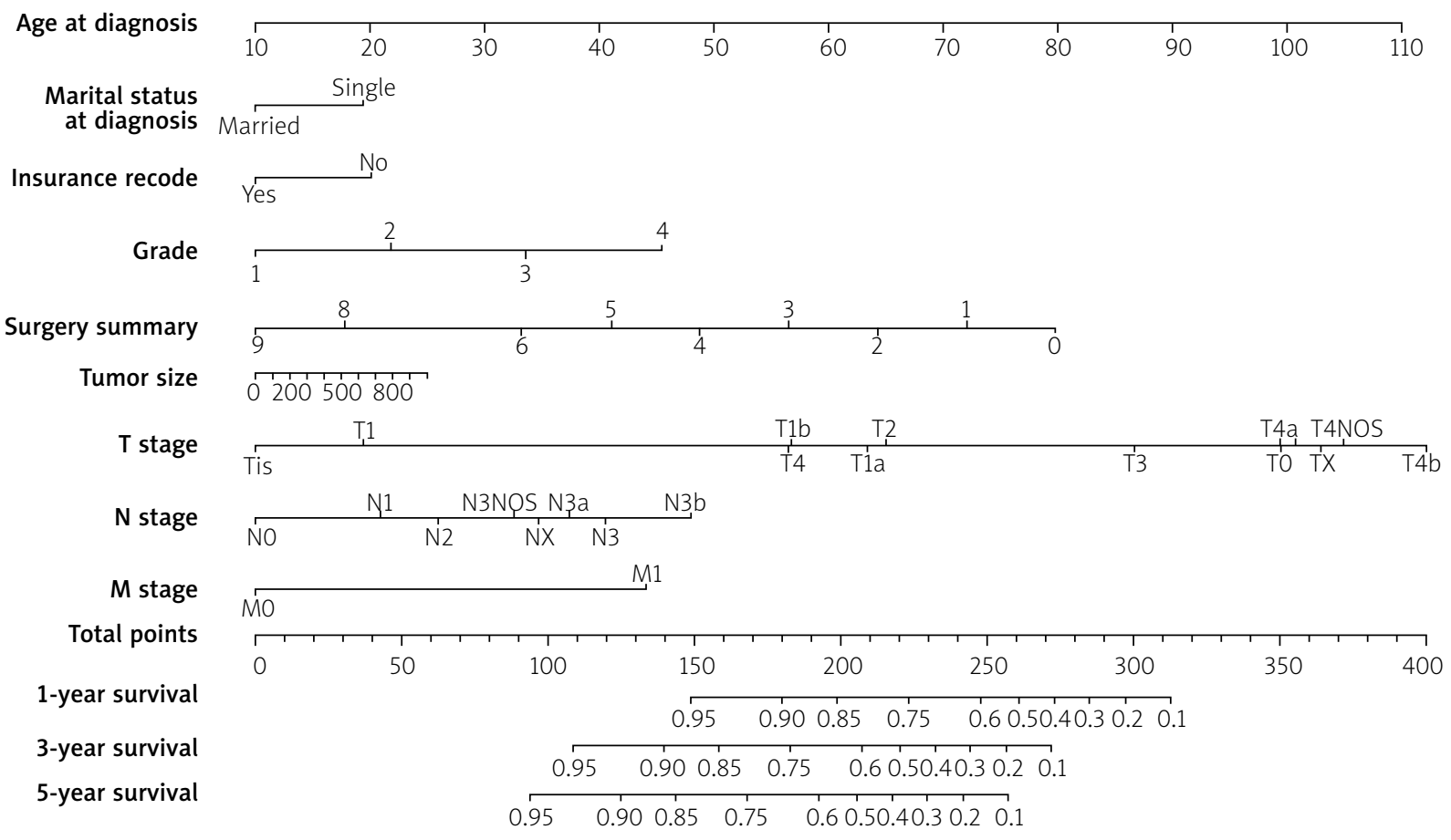

Figure 2. Nomogram of cox regression model

the best, while among the excisional biopsy group it was the worst (Figure $6 \mathrm{C}$ ). For local tumour excision surgery, except laser excision, the operation method has little effect on survival, but among the electrocautery group it was worse (Figure $6 \mathrm{D}$ ). In gastrectomy (partial, subtotal, hemi-) surgery, the survival of gastrectomy, NOS (partial, subtotal, hemi-), and lower (distal) gastrectomy (partial, subtotal, hemi-) group was worse than the antrectomy, lower (distal-less than $40 \%$ of stomach) and upper (proximal) gastrectomy (partial, subtotal, hemi-) group (Figure $6 \mathrm{E}$ ). Survival of the total gastrectomy group was the worst in the near-total or total gastrectomy surgery groups (Figure $6 \mathrm{~F}$ ), and the survival of partial or subtotal gastrectomy was the worst in gastrectomy, with removal of a portion of oesophagus surgery (Figure $6 \mathrm{G}$ ). In the gastrectomy with a resection in continuity with the resection of other organs surgery, the survival of gastrectomy with a resection in continuity with the resection of other organs, NOS and radical gastrectomy, in continuity with the resection of other organs group was the best, and near total or total gastrectomy, in continuity with the resection of other organs was worse, while partial or subtotal gastrectomy, in continuity with the resection of other organs group was the worst (Figure $6 \mathrm{H}$ ).

\section{Discussion}

The incidence of gastric cancer has been steadily declining since the early part of the $20^{\text {th }}$ century. However, due to the increase in life expectancy, the number of patients with gastric cancer continues to increase, especially among the elderly [5]. However, there are few studies on the choice of surgery for gastric cancer. Through 14,507 items of patient data from the SEER database, we used Cox multivariate regression model to explore the factors that affect the survival status of patients with gastric cancer, and came to the conclusion that age is the most important factor affecting the survival of patients, followed by the type of surgery, and histological grade, but these factors can not affect the survival of patients alone. For instance, advancing age is not an independent predictor of mortality following gastric surgery. Non-elective admission, pre-existing hypertension, valvular disease, and anaemia independently predicted increased morbidity and mortality following gastric surgery and should be carefully considered in 

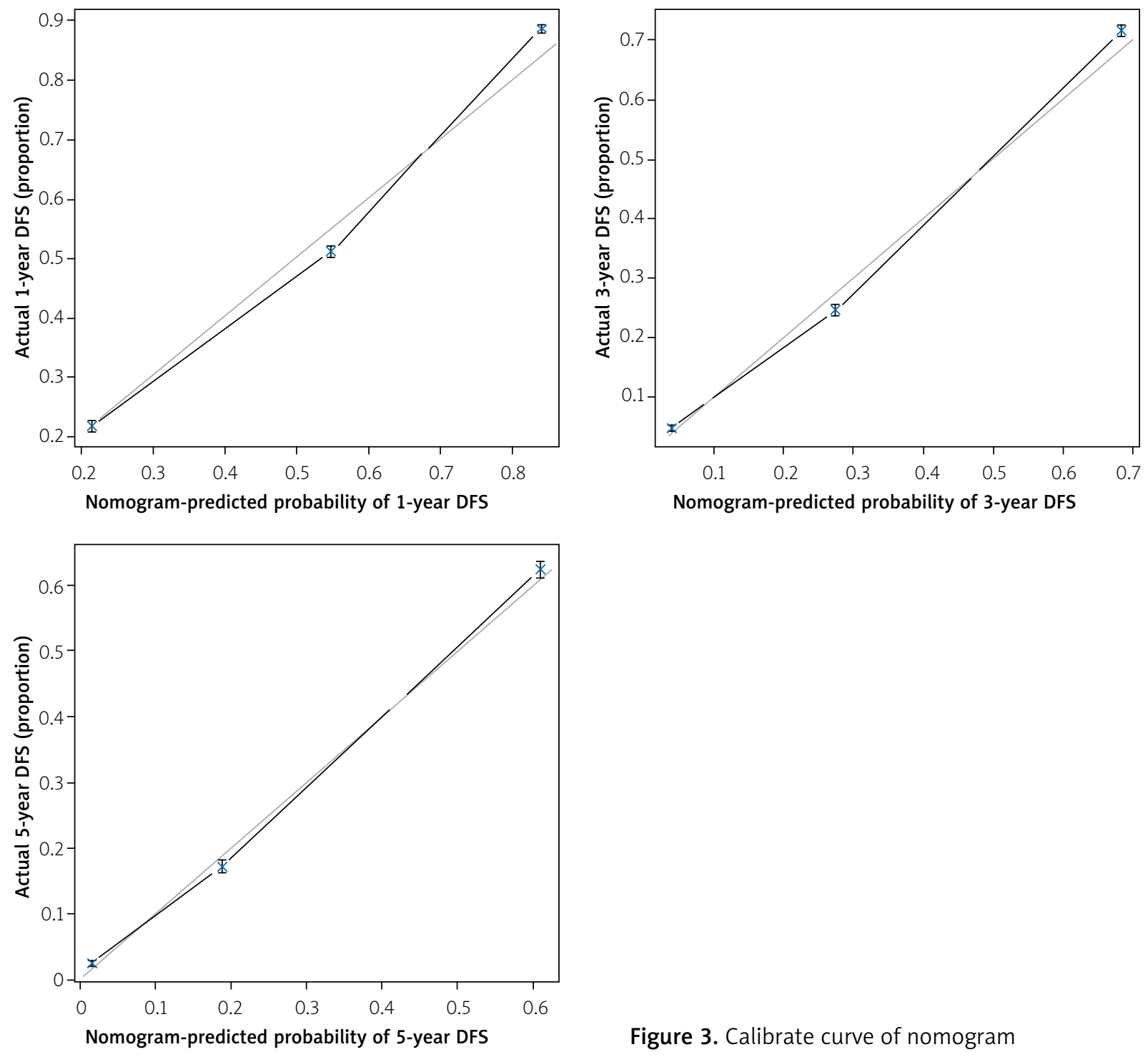

Figure 3. Calibrate curve of nomogram

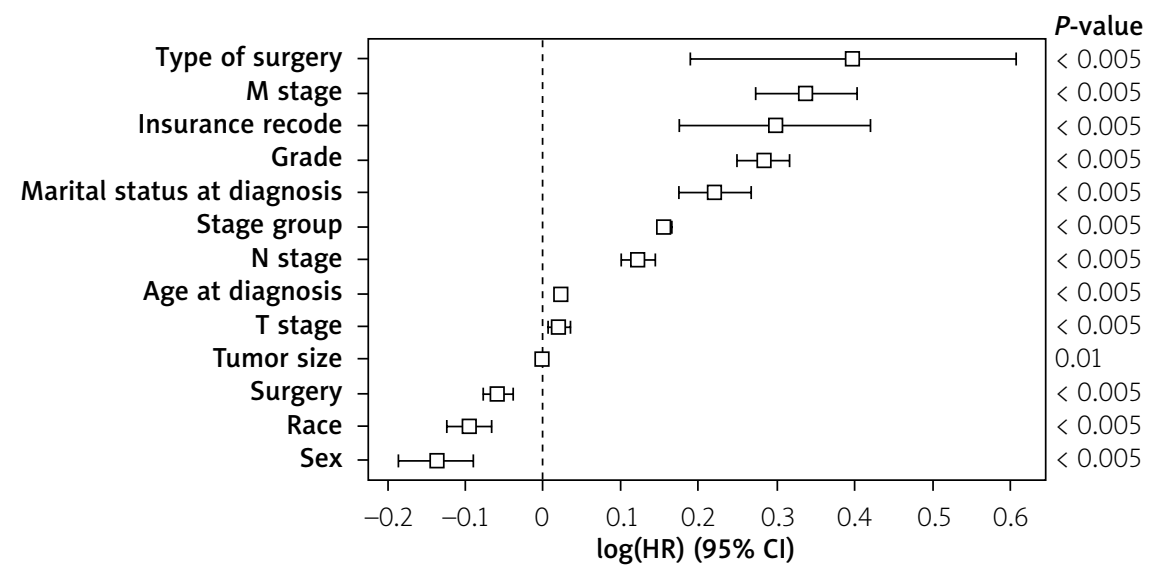

Figure 4. Multivariate analysis for factors affecting primary gastric cancer survival 
A

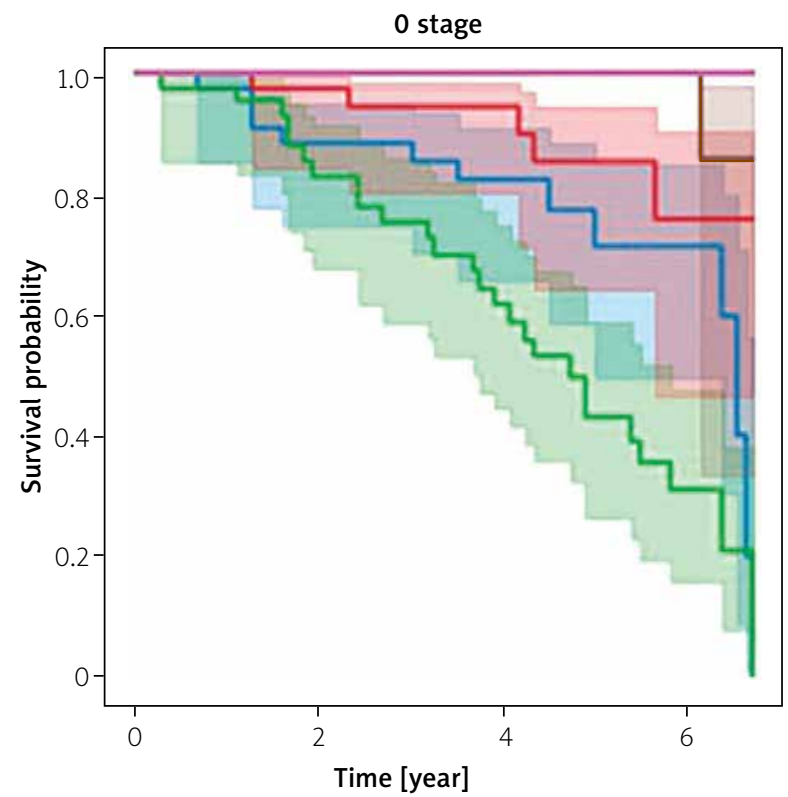

C

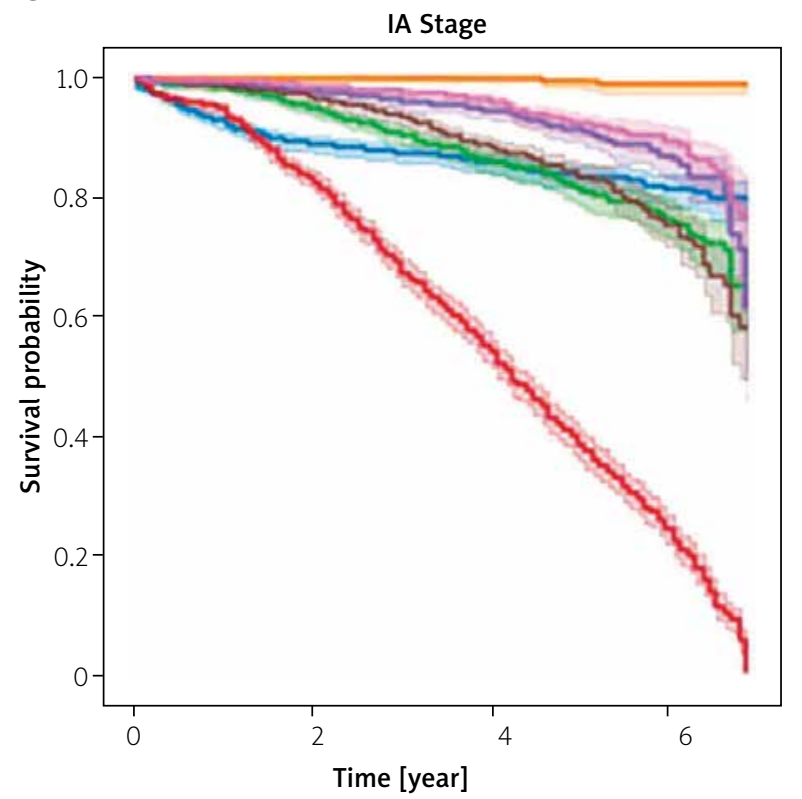

B

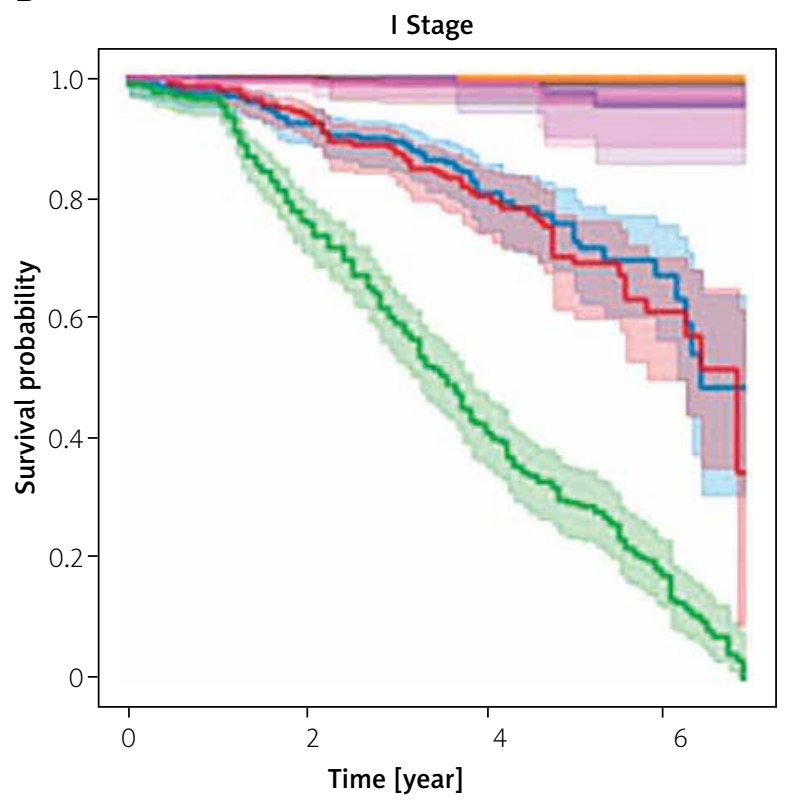

D

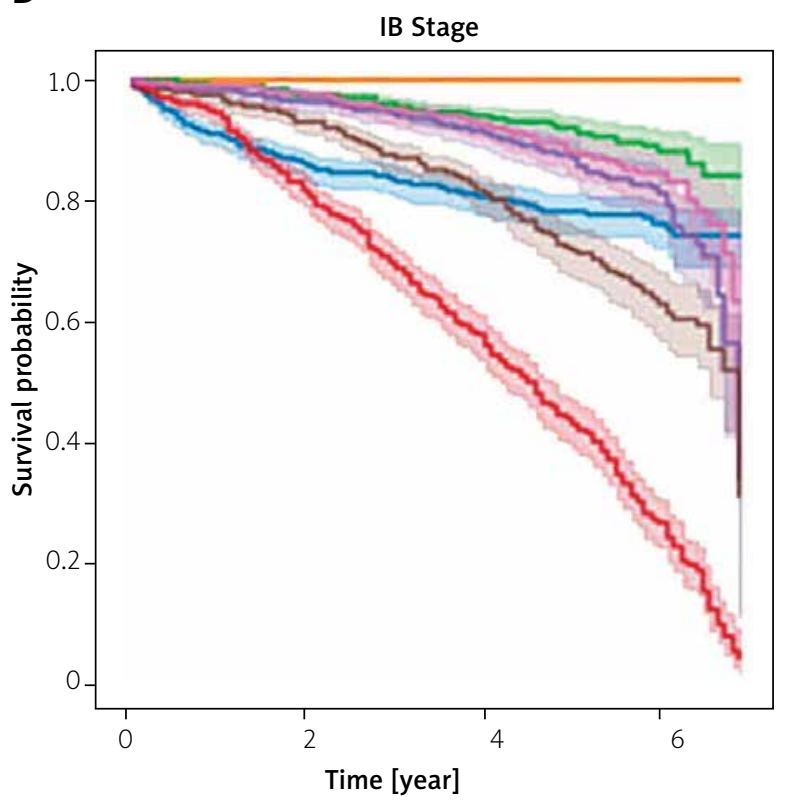

— No surgery of primary site - Local tumor destruction - Local tumor excision - Gastrectomy (partial, subtotal, hemi-) - Near-total or total gastrectomy _ Gastrectomy, NOS with removal of a portion of esophagus - Gastrectomy with a resection in continuity with the resection of other organs

Figure 5. Kaplan-Meier curve of AJCC $7^{\text {th }}$ ed. and type of surgery 
$E$

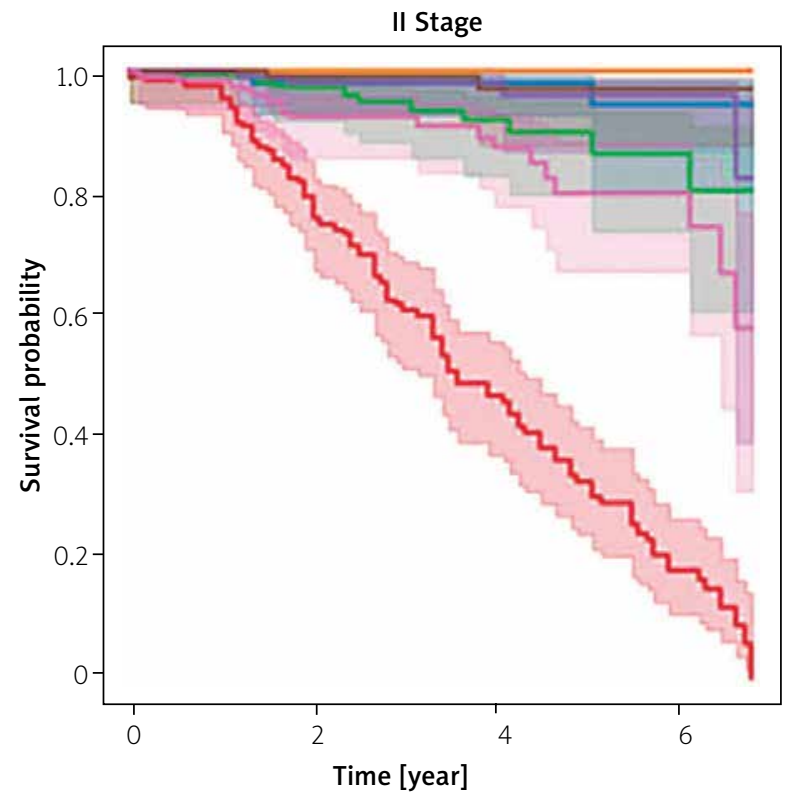

G

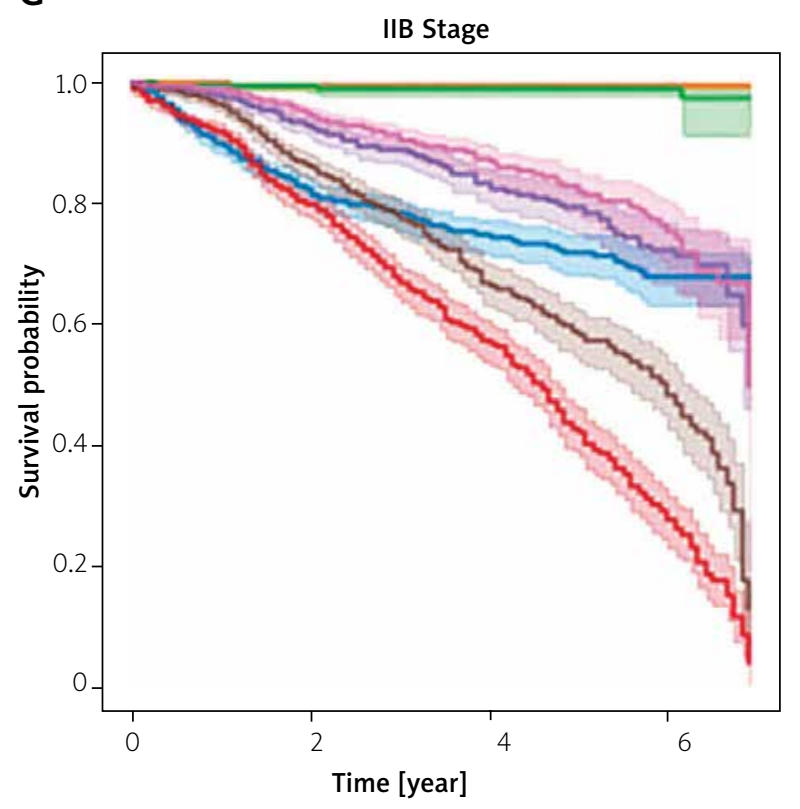

$\mathrm{F}$

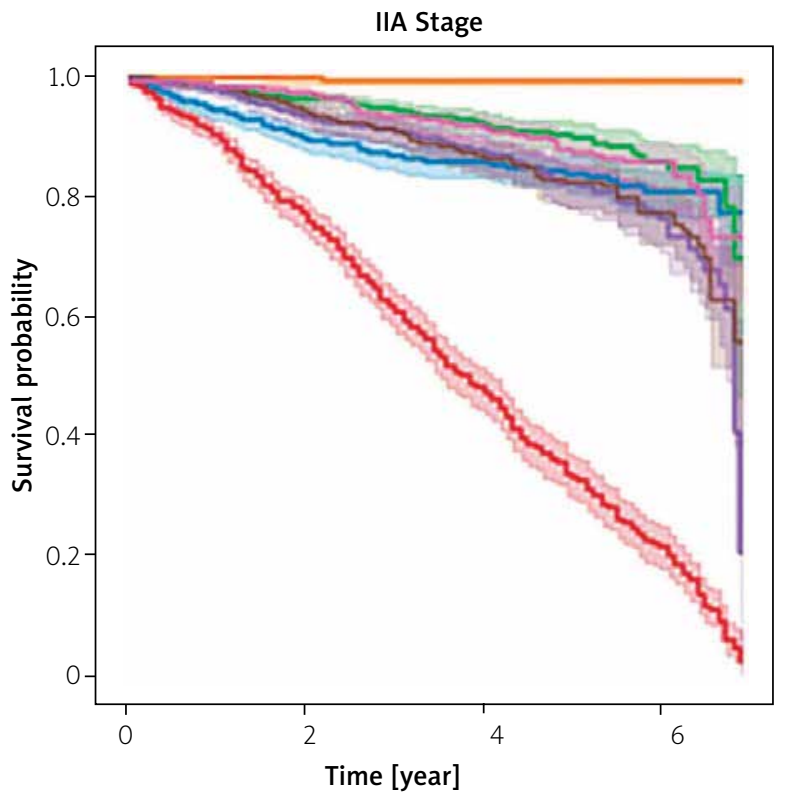

$\mathrm{H}$

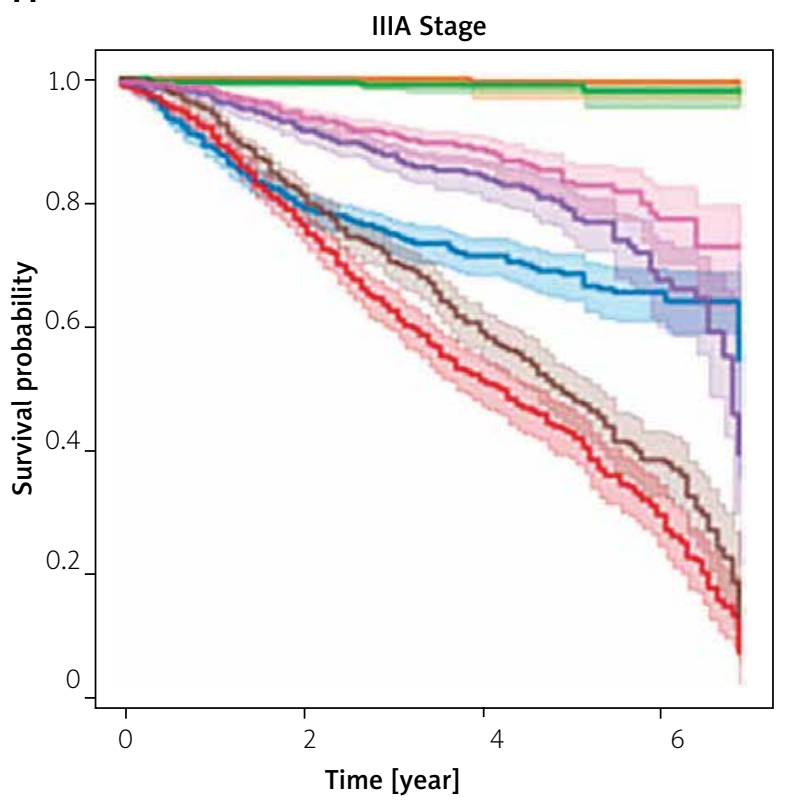

- No surgery of primary site _ Local tumor destruction _ Local tumor excision _ Gastrectomy (partial, subtotal, hemi-) - Near-total or total gastrectomy _ Gastrectomy, NOS with removal of a portion of esophagus - Gastrectomy with a resection in continuity with the resection of other organs

Figure 5. Cont. 
I

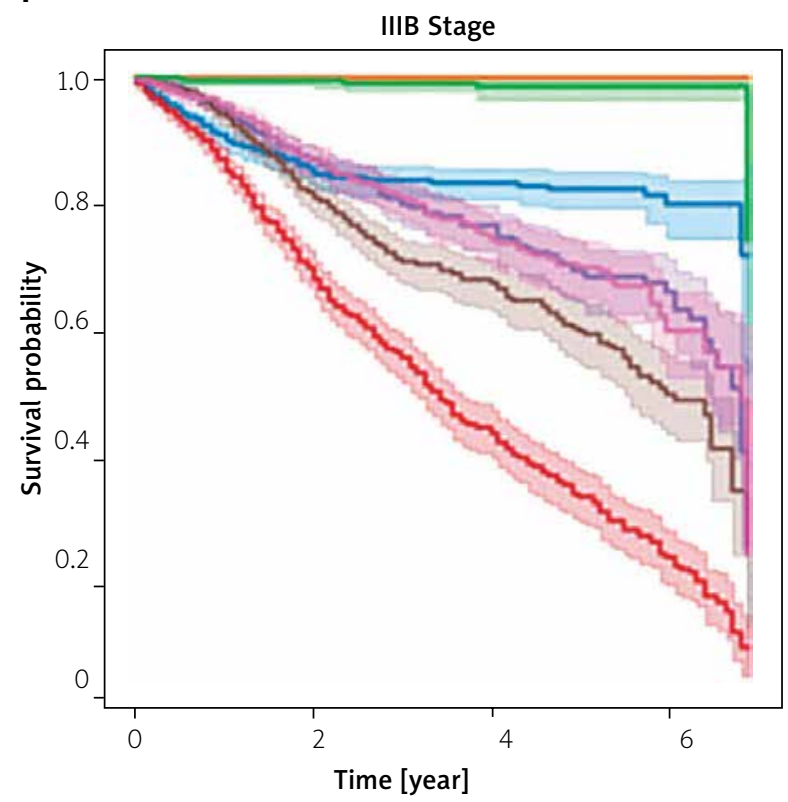

K

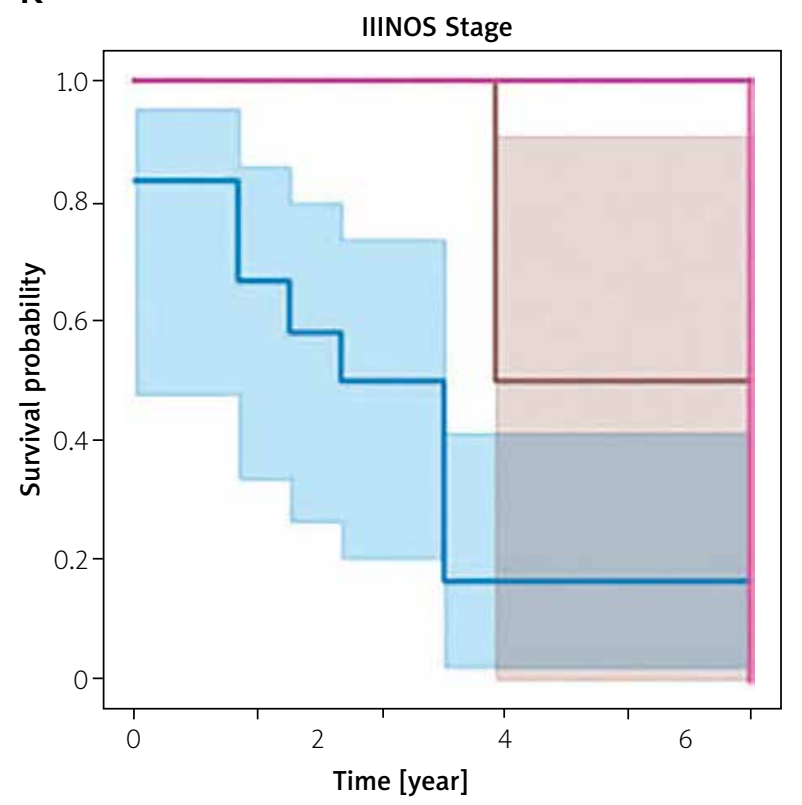

J

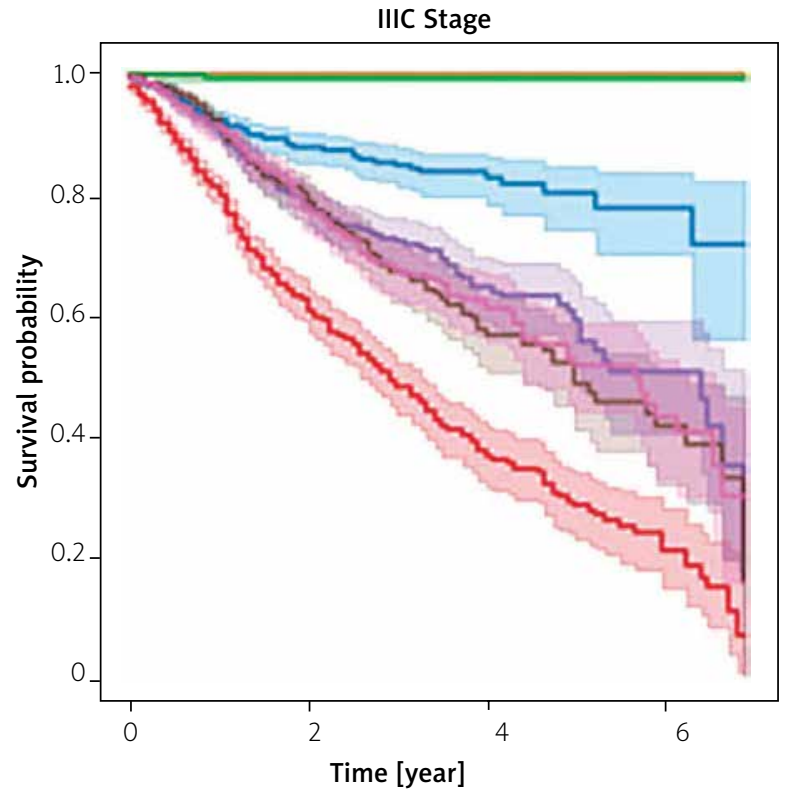

$\mathbf{L}$

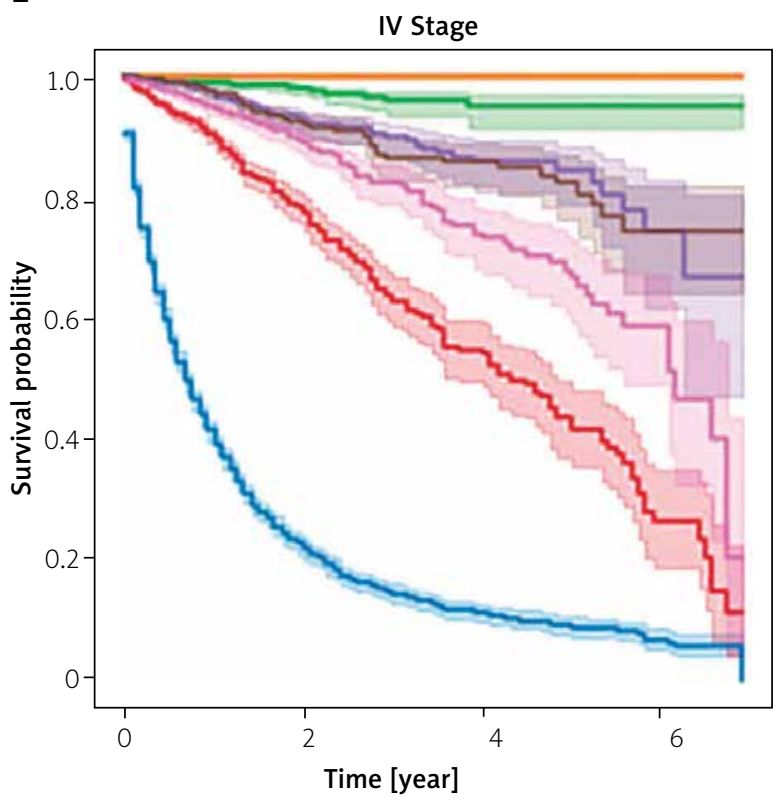

— No surgery of primary site _ Local tumor destruction - Local tumor excision — Gastrectomy (partial, subtotal, hemi-) - Near-total or total gastrectomy _ Gastrectomy, NOS with removal of a portion of esophagus - Gastrectomy with a resection in continuity with the resection of other organs

Figure 5. Cont. 
A

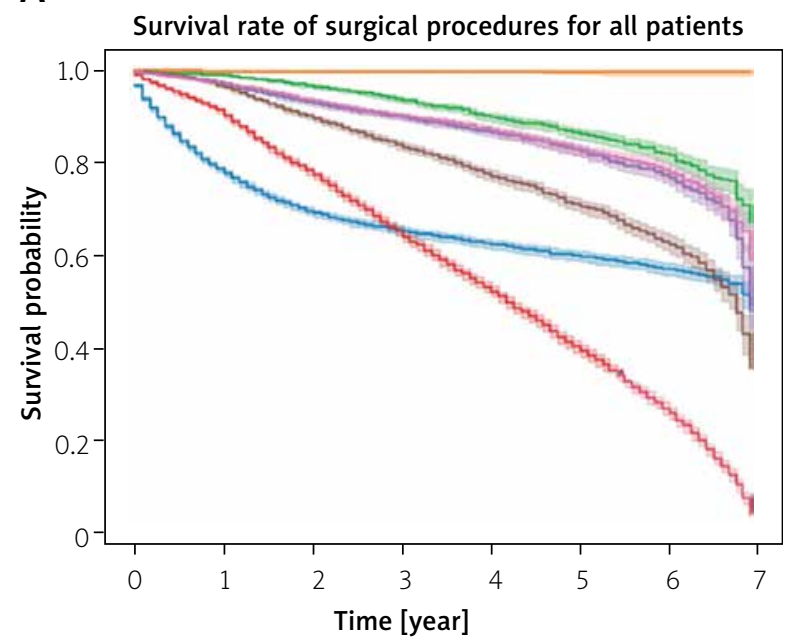

- No surgery of primary site

- Local tumor destruction

- Local tumor excision

- Gastrectomy (partial, subtotal, hemi-)

- Near-total or total gastrectomy

- Gastrectomy, with removal of a portion of esophagus

- Gastrectomy with a resection in continuity with the resection

$$
\text { of other organs }
$$

C

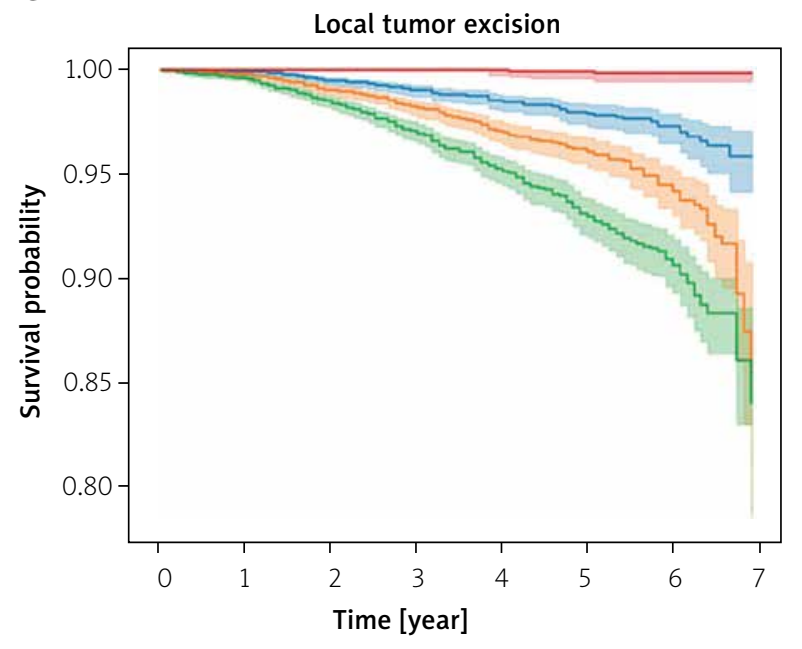

- Local tumor excision, NOS

- Polypectomy

- Excisional biopsy

- Laser excision
B

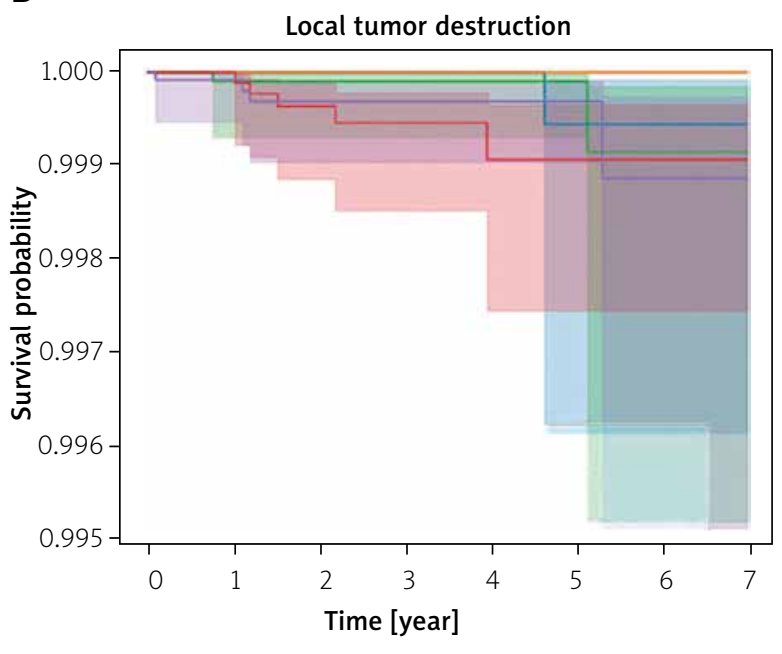

- Local tumor destruction, NOS

- Photodynamic therapy (POT)

- Electrocautery, fulguration (includes hot forceps for tumor

destruction)
- Cryosurgery

- Laser

D Any combination of local tumor excision, NOS

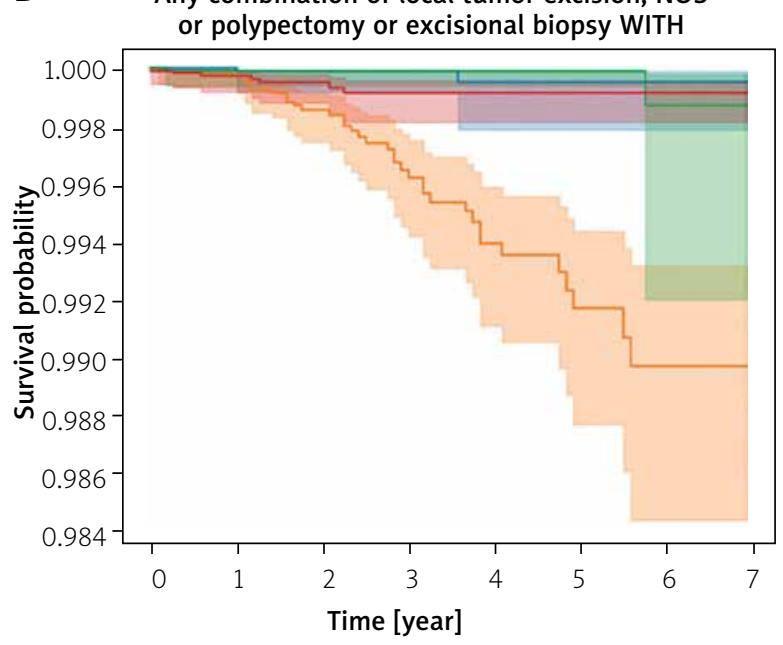

- Photodynamic therapy (POT)

- Electrocautery

- Cryosurgery

- Laser ablation

Figure 6. Kaplan-Meier curve of type of surgery and surgical methods 
$E$

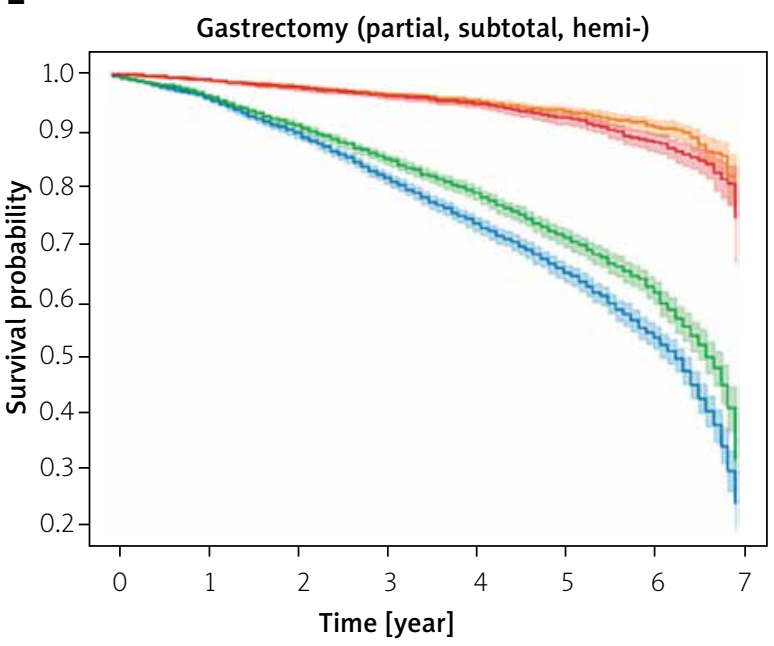

- Gastrectomy, NOS (partial, subtotal, hemi-)

- Antrectomy, lower (distal-less than $40 \%$ of stomach)

- Lower (distal) gastrectomy (partial, subtotal, hemi-)

- Upper (proximal) gastrectomy (partial, subtotal, hemi-)

G

Gastrectomy, with removal of a portion of esophagus

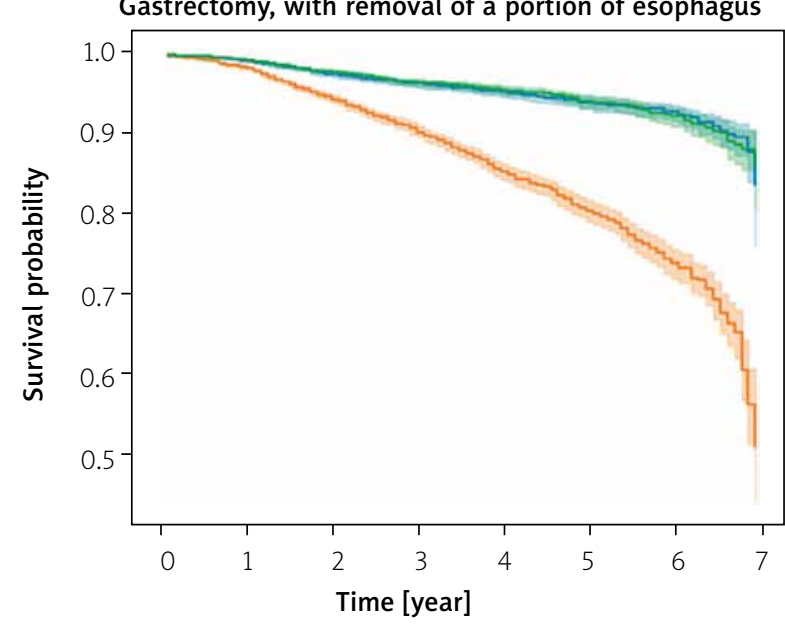

- Gastrectomy, NOS WITH removal of a portion of esophagus

- Partial or subtotal gastrectomy

- Near total or total gastrectomy

Figure 6. Cont.

surgical planning and counselling [6-8]. In this process, we also found some interesting results. For example, men are at a higher risk of developing gastric cancer than women; this result is consistent with the mainstream research showing that androgen receptor may be responsible for the gender disparity in gastric cancer [9]. Race also affects the prognosis of patients with gastric cancer to some extent; the same conclusion was
F

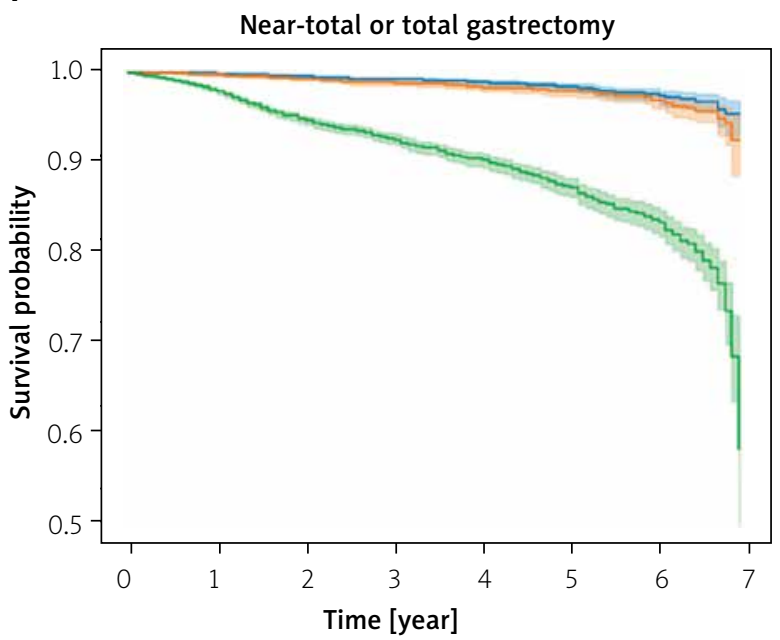

- Near-total or total gastrectomy, NOS

- Near-total gastrectomy - Total gastrectomy

$\mathrm{H} \quad$ Gastrectomy with a resection in continuity with the resection of other organs

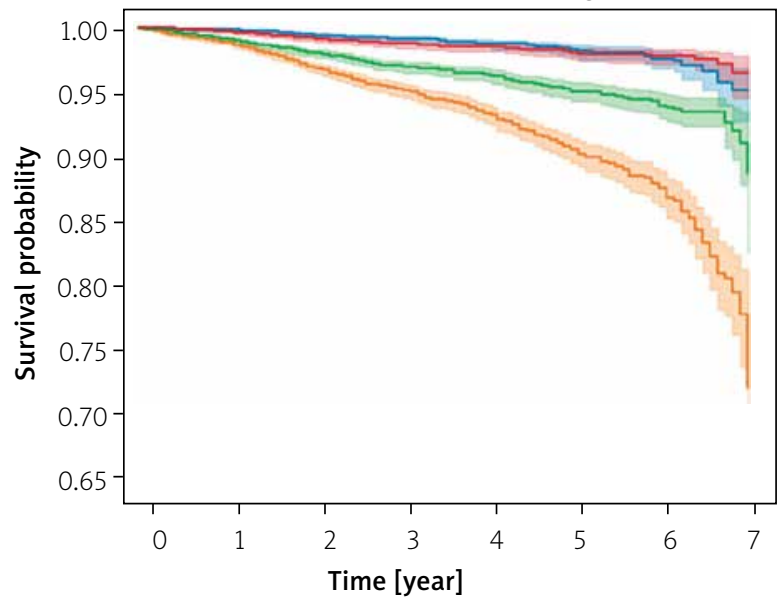

- Gastrectomy with a resection in continuity with the resection of other organs, NOS

- Partial or subtotal gastrectomy, in continuity with the resection of other organs

- Near total or total gastrectomy, in continuity with the resection of other organs - Radical gastrectomy, in continuity with the resection of other organs

drawn from other studies [10,11]. Marital status and health insurance status also affect the prognosis of patients to some extent. The above conclusions are drawn from the nomogram (Figure 2). According to the type of surgery and the AJCC $7^{\text {th }}$ ed. stage of the patients, the group experiment was carried out, and the Kaplan-Meier model was used to compare the survival rate of the patients, so as to judge the effect of the type of surgery 
on the survival benefit. The most striking conclusion is that gastrectomy (partial, subtotal, hemi-) is the worst type of surgery, especially in patients with intermediate and advanced gastric cancer. Gastrectomy NOS (partial, subtotal, hemi-) is the worst surgical method in patients with gastric cancer who undergo gastrectomy (partial, subtotal, hemi-). Three surgical methods: partial gastrectomy, Billroth I, and Billroth II, are included in the gastrectomy NOS (partial, subtotal, hemi-) group. Because there are no detailed records of Billroth I and Billroth II in the SEER database, it is generally believed that the Billroth II method of anastomosis is associated with higher rate of early postoperative complications, and the Billroth I method should be the first choice after a distal gastrectomy as long as the anatomic and oncological environment of an individual patient allows it [12]. In other radical operations, the effect of gastrectomy with a resection in continuity with the resection of other organs is the best, but the effect of AJCC $7^{\text {th }}$ ed. $\checkmark$ stage is the worst except Gastrectomy (partial, subtotal, hemi-). Among the conclusions of the study, there are also some that are contrary to the existing research conclusions. For example, near-total gastrectomy is not a better option for upper-third early gastric cancer than total gastrectomy [13], but in this study, the survival benefit of near-total gastrectomy was better than that of total gastrectomy (Figure $6 \mathrm{~F}$ ). In palliative surgery, local tumour destruction and local tumour excision are both safe and effective treatments for early gastric cancer, and it is useful for histological confirmation of successful treatment [14]. Lastly, we cannot control the quality of primary data, and pathological diagnoses from multiple hospitals will lead to inevitable bias.

\section{Acknowledgments}

This work is funded by the National University Student Innovation and Entrepreneurship Training Program of P.R. China under Grant 202010373032 and Grant S201910373149, and the Natural Science Research Project of the Education Department of Anhui Province of P.R. China under Grant KJ2019B05 and Grant KJ2017A390.

\section{Conflict of interest}

The authors declare no conflict of interest.

\section{References}

1. https://www.wcrf.org/ Last accessed on 29/4/2020.

2. Gallo M, Altieri F, Stella Di Stadio C, et al. An overview on factors underlying gastric cancer; strategies for its management with particular reference to diet. J Gastrointestinal Dig System 2016; 6: 1-10.
3. Amin MB, Greene FL, Greene FL, et al. The Eighth Edition AJCC Cancer Staging Manual: Continuing to build a bridge from a population-based to a more "personalized" approach to cancer staging. CA Cancer J Clin 2017; 67: 93-9.

4. Surveillance, Epidemiology and End Results Program. About the SEER Program. Available at: http://seer.cancer.gov/about. Accessed May 1.

5. Orsenigo E, Tomajer V, Saverio Di P, et al. Impact of age on postoperative outcomes in 1118 gastric cancer patients undergoing surgical treatment. Gastric Cancer 2007; 10: 39-44.

6. Karkar A, Patil S, Chamberlain RS. A population-based outcomes analysis of the impact of age on morbidity and mortality following gastrectomy: an analysis of 13,799 patients from the nationwide inpatient sample database. J Cancer Therapy 2013; 4: 80-91.

7. Yang D, Hendifar A, Lenz C, et al. Survival of metastatic gastric cancer: significance of age, sex and race/ethnicity. J Gastrointest Oncol 2011; 2: 77-84.

8. Coleman KJ, Brookey J. Gender and racial/ethnic background predict weight loss after Roux-en-Y gastric bypass independent of health and lifestyle behaviors. Obes Surg 2014; 24: 1729-36.

9. Tian $\mathrm{Y}$, Wan $\mathrm{H}$, Lin $\mathrm{Y}$, et al. Androgen receptor may be responsible for gender disparity in gastric cancer. Med Hypotheses 2013; 80: 672-4.

10. Merchant SJ, Li L, Kim J. Racial and ethnic disparities in gastric cancer outcomes: more important than surgical technique? World J Gastroenterol 2014; 20: 11546-51.

11. Yao JC, Schnirer II, Reddy S, et al. Effects of sex and racial/ethnic group on the pattern of gastric cancer localization. Gastric Cancer 2002; 5: 208-12.

12. Sah BK, Chen MM, Yan M, Zhu ZG. Gastric cancer surgery: Billroth I or Billroth II for distal gastrectomy? BMC Cancer 2009; 9: 428.

13. An JY, Youn HG, Choi MG, et al. The difficult choice between total and proximal gastrectomy in proximal early gastric cancer. Am J Surg 2008; 196: 587-91.

14. Oka S, Tanaka S, Kaneko I, et al. Endoscopic submucosal dissection for residual/local recurrence of early gastric cancer after endoscopic mucosal resection. Endoscopy 2006; 38: 966-1000.

Received: 12.07 .2020

Accepted: 10.08 .2020 